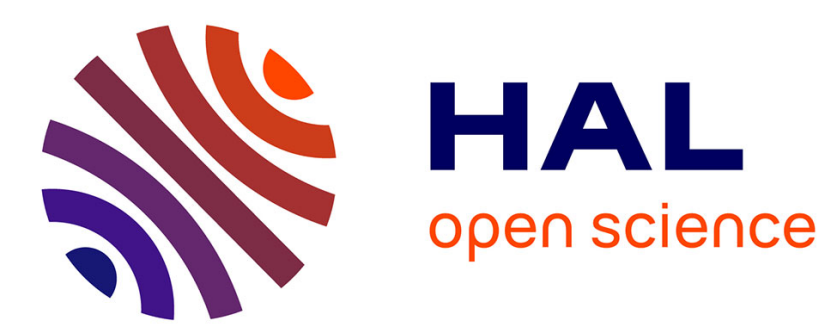

\title{
Effets des transformations de phase sur la tenue mécanique à haute température des céramiques réfractaires
}

Nicolas Schmitt, Poirier Jacques

\section{- To cite this version:}

Nicolas Schmitt, Poirier Jacques. Effets des transformations de phase sur la tenue mécanique à haute température des céramiques réfractaires. Matériaux \& Techniques, 2009, 97, pp.41 - 49. 10.1051/mattech/2010006 . hal-01713971

\section{HAL Id: hal-01713971 \\ https://hal.science/hal-01713971}

Submitted on 21 Feb 2018

HAL is a multi-disciplinary open access archive for the deposit and dissemination of scientific research documents, whether they are published or not. The documents may come from teaching and research institutions in France or abroad, or from public or private research centers.
L'archive ouverte pluridisciplinaire $\mathbf{H A L}$, est destinée au dépôt et à la diffusion de documents scientifiques de niveau recherche, publiés ou non, émanant des établissements d'enseignement et de recherche français ou étrangers, des laboratoires publics ou privés. 


\title{
Effets des transformations de phase sur la tenue mécanique à haute température des céramiques réfractaires Influence of phase transformations on the mechanical behaviour of refractory ceramics at high temperature
}

\author{
Nicolas SCHMITT ${ }^{1,2}$, Jacques POIRIER ${ }^{3,4}$ \\ ${ }^{1}$ LMT-Cachan (ENS de Cachan, UMR 8535 CNRS, UPMC), 61 av. du Président Wilson, F-94235 \\ Cachan (France) \\ ${ }^{2}$ IUFM de Créteil (Université Paris-Est Créteil), Place du 8 mai 1945, 93203 Saint Denis (France) \\ ${ }^{3}$ CNRS-CEMHTI, 1D, avenue de la Recherche Scientifique, 45071 Orléans Cedex 2 (France) \\ ${ }^{4}$ Polytech', Université d'Orléans, 8 rue L. de Vinci, 45072 Orléans Cedex 2 (France)
}

Email : Nicolas.Schmitt@lmt.ens-cachan.fr \& jacques.poirier@cnrs-orleans.fr

Résumé :

Les céramiques réfractaires, utilisées à haute température, sont soumises à d'intenses sollicitations thermochimiques et thermomécaniques. La maîtrise de leur microstructure et des transformations de phases qu'elles subissent en service est essentielle pour assurer une bonne tenue mécanique des structures réfractaires. De grands progrès ont été réalisés : l'association de techniques expérimentales performantes avec de puissants outils numériques (calculs thermochimiques et thermomécaniques) permet aujourd'hui d'obtenir des informations inaccessibles de manière directe (e.g., phénomènes d'endommagement). De même, les outils de modélisation multiphysique et multi-échelle permettent de développer des matériaux plus performants résistant à de plus hautes températures et de manière plus durable. Dans ce papier on présente certaines interactions entre le comportement mécanique et les transformations de phases de ces céramiques, les moyens développés pour caractériser leur comportement, les techniques d'investigation utilisées pour accéder à leur état microstructural in situ et identifier leur évolution au cours du temps. On expose également quelques démarches mises en œuvre et certains outils développés au cours de ces dernières années, aboutissant aujourd'hui à une meilleure connaissance du comportement en service des céramiques réfractaires et à l'identification de paramètres concernant les matériaux et/ou les procédés permettant d'allonger leur durée de vie.

\section{Abstract:}

Refractories used at high temperature are subjected to high chemical and mechanical stresses. The mastery of their microstructure as well as the phase changes occurring in service is essential to ensure resistance to wear and failure of refractory linings. Great progress has been made: combining efficient techniques for the investigation of the microstructure with powerful numerical tools (thermo-chemical and thermo-mechanical computations) provides information (e.g., degradation mechanisms) that cannot be obtained directly. Also multi-physical and multi-scale models developping materials with high-performance for higher temperature and with longer life-time.

In this paper, through several examples show some interactions between the mechanical behavior and the microstructure transformations of refractory ceramics. The tools developed to characterize their microstructure change in situ (e.g., at high temperature) and to identify their kinetics are described. Some methodologies and tools developed in recent years, today, provide a better understanding of inservice behavior of refractories while identifying the critical material and process parameters likely to increase life-time. 
Mots clés : céramique réfractaire ; comportement thermomécanique ; corrosion ; transformation de phase ; haute température ; couplages multi-physique ; couplages multi-échelle

Key words : refractory ceramics; thermo-mechanical behavior; corrosion; phase changes; high temperature; multi-physic analysis; multi-scale analysis

\section{1) Introduction}

Dans l'industrie, les céramiques réfractaires sont utilisées pour confiner l'énergie et faciliter l'élaboration des matériaux (métaux, ciment, verres, céramiques, ...) à hautes températures [13]. Matériaux de grande diffusion, ces céramiques structurales sont essentielles pour garantir le développement de nouvelles technologies. Consciente du rôle stratégique de ces produits et de leur incidence dans le domaine de l'innovation et de la compétitivité, depuis quelques années les grands groupes (sidérurgie, verrerie, métallurgie des non ferreux, énergie, environnement) et certains grands producteurs de matériaux réfractaires s'associent à des laboratoires de recherches pour développer leur connaissance sur ces matériaux. En France, plusieurs projets nationaux en cours ou réalisés $[5,8]$ attestent de la vitalité de la recherche française dans ce domaine.

Les céramiques réfractaires sont soumises à d'intenses sollicitations thermochimiques et thermomécaniques. Ces sollicitations souvent associées se produisent dans un environnement difficile à reproduire en laboratoire. Leurs effets sur la durée de vie des réfractaires sont donc singulièrement difficiles à évaluer de manière quantitative. La maîtrise de la composition et de l'architecture des matériaux à l'échelle de la microstructure demeure un enjeu essentiel fondamental pour assurer une bonne tenue des revêtements et des structures réfractaires, mais aussi satisfaire les exigences environnementales de plus en plus sévères [18].

Pour relever les futurs défis auxquels ces industries sont confrontées (élaboration de matériaux avec moins d'impuretés, conception de barrières de sécurité dans les centrales nucléaires de génération IV, réalisation de réacteurs de gazéification de la biomasse, ...), une connaissance plus fine des transformations de phases et de leur interaction avec la mécanique est requise. Ces transformations sont inévitables compte tenu des niveaux élevés de température en service. Elles sont souvent considérées comme préjudiciables, par exemple en accélérant la corrosion, en réduisant de manière dramatique la résistance mécanique. Elles peuvent aussi avoir un effet positif sur la tenue en service des pièces réfractaires, par exemple, en rendant le matériau - quasi-fragile à basse température - plus ductile et visqueux à haute température, de sorte qu'il supporte mieux les déformations et contraintes thermiques, ou encore en développant des barrières «microstructurales « in situ » pour limiter la pénétration des agents corrosifs [15].

Les progrès réalisés ces dernières années dans les techniques expérimentales $[5,10,12,17]$ et les méthodes numériques dans les domaines des calculs thermochimiques et thermomécaniques $[2,3,9]$ sont importants. Les analyses couplant expérience et modélisation permettent aujourd'hui d'obtenir des informations inaccessibles auparavant ou par des moyens d'observation plus classiques d'échantillons post-mortem. La modélisation multiphysique et multi-échelle [16,21] et les collaborations scientifiques entre équipes de recherche pluridisciplinaire permettent aujourd'hui d'avoir une meilleure compréhension des phénomènes d'endommagement couplés de type «thermique-corrosion-mécanique » et le développement des matériaux plus performants, résistant à des températures plus élevées et de manière plus durable.

Après avoir illustré quelques modes de rupture observés dans ces matériaux, nous montrerons sur quelques exemples ciblés, les méthodes pouvant être mises en œuvre pour mieux comprendre les dégradations d'origine multi-physique observées, et qui sont nécessaires pour proposer des solutions d'amélioration de la tenue en service. 


\section{2) Dégradations des pièces et des revêtements réfractaires}

On ne peut fournir ici une vision exhaustive des multiples dégradations observées dans les pièces et les garnissages réfractaires. La variété des céramiques est en effet très importante. Par exemple, dans une usine sidérurgique, il est courant de répertorier plusieurs centaines de matériaux réfractaires le long de la chaîne de production de l'acier. Le choix du matériau est adapté à l'environnement agressif dans lequel il est placé. Ainsi, une céramique structurale utilisée dans un convertisseur (transformation de la fonte en acier) possède des caractéristiques physiques et mécaniques différentes de celle présente dans une poche à acier (traitement de métallurgie secondaire de l'acier). A l'échelle d'un même «outil sidérurgique », le garnissage est déjà un revêtement composite complexe (multi-couches et multimatériaux).

Les nombreuses expertises réalisées sur des matériaux dégradés in situ ou en laboratoire dans un environnement contrôlé ont révélé que la corrosion et les contraintes thermomécaniques constituent les principales causes de dégradation et d'usure de ces matériaux $[13,18]$. Sur cette base, il est aujourd'hui couramment fait référence aux trois grands pôles qui génèrent des sollicitations et gouvernent ces dégradations, i.e., Thermique, Mécanique et Chimie. Il y a encore une vingtaine d'années, les causes principales de dégradations étaient analysées au travers des pôles «Chimie-Thermique ». Améliorer la résistance à la corrosion du matériau suffisait pour garantir une augmentation notable des performances globales. Il est évident que cette approche n'est plus suffisante aujourd'hui comme l'illustrent les propositions d'explication des causes de dégradation présentées ci-dessous.

\section{1) Dégradations des garnissages de béton réfractaire au cours du séchage}

A des températures assez basses - typiquement inférieures à $600^{\circ} \mathrm{C}$-, les céramiques réfractaires contenant des liants hydrauliques subissent déjà des dommages lors de la phase de séchage, soit en usine ou in situ, soit suite à des transformations microstructurales (déshydratation du liant) soit à des pressions de vapeur excessives. Par exemple, la figure 1 montre l'état de fissuration du revêtement de durée en béton réfractaire sur la périphérie d'une poche à acier observée à mi hauteur sur une bande d'environ un mètre. Un premier réseau de fissures horizontales et verticales s'est développé après le traitement thermique qui a pour but d'éliminer l'eau libre et de constitution. Le réseau se densifie au fur et à mesure des cycles d'affinage de l'acier.

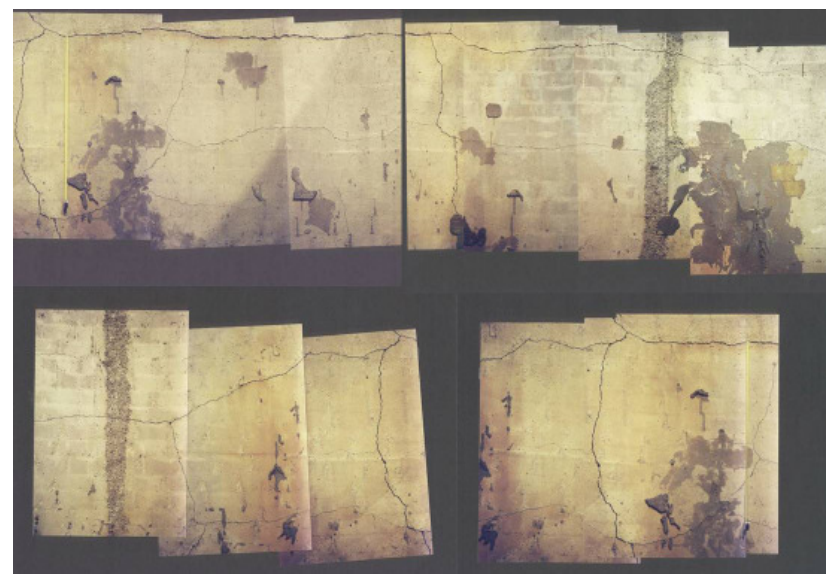

Fig. 1. Réseau de fissures développé dans un revêtement réfractaire de durée d'une poche à acier observé lors la première réfection de la couche d'usure [22]

\section{Fig. 1. Pattern of cracks observed in the safety refractory lining of a steel ladle after the first replacement of the working lining [22]}

La diminution de la résistance mécanique du béton réfractaire et le retrait irréversible apparaissant lors de la déshydratation sont des facteurs qui contribuent à l'apparition de ces dégradations. Les contraintes sont moins élevées dans le cas des bétons à très faible teneur en ciment alumineux, ce qui indique que les contraintes thermiques ne suffisent pas à justifier ces endommagements. 
On notera ici que les dégradations apparaissent suite à une élévation de température. Les transformations de phases observées se produisent en dehors d'une interaction chimique entre le matériau et son environnement, ce qui n'est pas le cas des exemples suivants.

\section{2) Dégradations du creuset des haut fourneaux}

La transformation du minerai de fer et du coke métallurgique en fonte s'effectue dans un hautfourneau. Le revêtement de ce réacteur dans la zone basse, dont la température dépasse $1500^{\circ} \mathrm{C}$, est réalisé par une maçonnerie de blocs de réfractaires de carbone dont la durée de vie moyenne est d'une vingtaine d'années. Dans la zone du creuset, sur les pièces usées, on observe en profondeur une zone dégradée (la brittle layer) avec des fissures parallèles à la face chaude et de la matière désagrégée (Fig. 2).

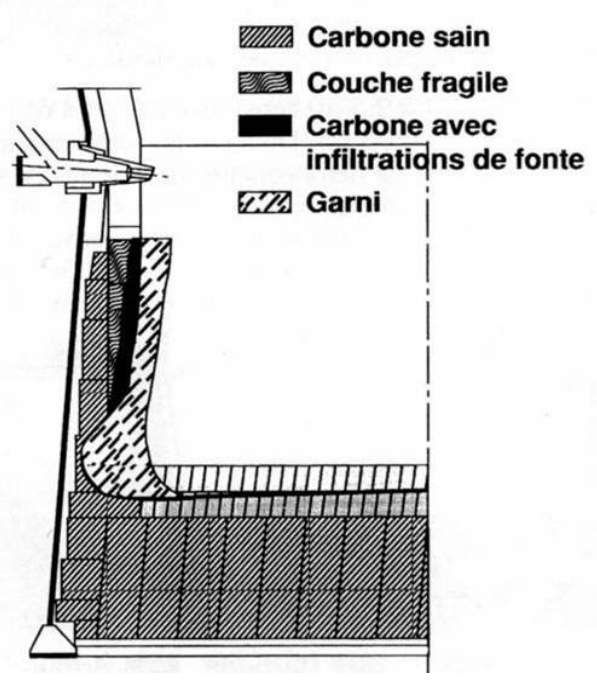

a) Garnissage du fond

a) Bottom lining

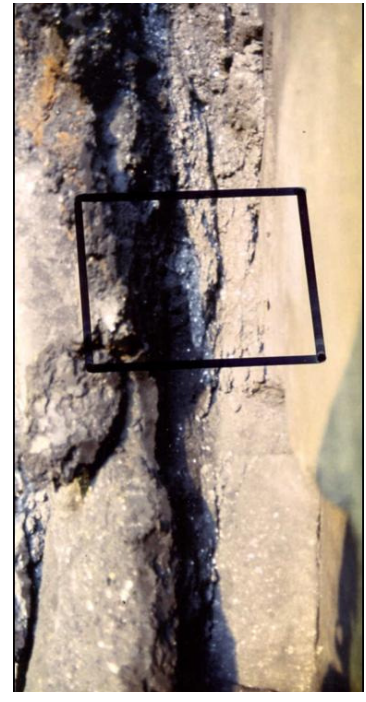

b) Fissuration du garnissage

b) Fracture of the lining

Fig. 2. Endommagement du réfractaire carboné dans le creuset du haut-fourneau

Fig. 2. Damage of a carbon refractory lining in the hearth of a blast furnace

Différentes causes ont été évoquées pour expliquer l'origine de cette fissuration en profondeur qui conduit à l'apparition d'une zone pulvérulente sur une épaisseur qui grossit au cours du temps. Des analyses post-mortem ont montré que de nouvelles phases étaient apparues $\left(\mathrm{ZnO} \cdot \mathrm{SiO}_{2}\right.$, $\mathrm{K}_{2} \mathrm{O} \cdot \mathrm{Al}_{2} \mathrm{O}_{3} \cdot 2 \mathrm{SiO}_{2}, \quad \mathrm{~K}_{2} \mathrm{O} \cdot \mathrm{Al}_{2} \mathrm{O}_{3} \cdot 4 \mathrm{SiO}_{2}$ ). L'explication aujourd'hui communément admise est la suivante : les phases gazeuses alcalines et des vapeurs métalliques présentes dans les charges pénètrent dans le réfractaire. Comme il existe un gradient de température dans le réfractaire, les gaz se condensent. Par exemple, du fait de la condensation du zinc et des alcalins à une température voisine de $900^{\circ} \mathrm{C}$, le contact avec les phases secondaires du réfractaire carboné $\left(\mathrm{SiO}_{2}\right.$ et $\left.\mathrm{Al}_{2} \mathrm{O}_{3}\right)$ produit par réaction des silico-aluminates de potassium et des silicates de zinc qui se déposent. La formation de ces phases s'accompagne d'une forte expansion volumique et d'un comportement mécanique moins ductile qui produit une microfissuration du volume.

L'endommagement apparaît dans une zone non exposée à la fonte liquide en contact avec le réfractaire. Il a lieu essentiellement par apport de matière qui interagit avec celle présente dans le matériau vierge. Ainsi, même si le volume élémentaire représentatif n'avait pas été contraint par la présence de contraintes thermiques, la désagrégation se serait produite.

\section{3) Dégradations causées par la corrosion couplée à la mécanique}

Il s'agit là d'une forme de dégradation très courante dans les réfractaires au contact des laitiers utilisés dans les outils sidérurgiques, des alcalins présents dans le verre en fusion des fours verriers, des déchets en cours de transformation dans les incinérateurs. 
L'exemple suivant illustre ce mécanisme [1,19]. La dalle d'impact située dans le fond d'une poche à acier est sollicitée de manière intense. Le réfractaire utilisé est généralement un béton alumineux contenant des particules fines de magnésie qui se combinent à haute température avec les fines d'alumine et d'aluminates de chaux pour former des spinelles alumine-magnésie - en proportion limitée, e.g. 10 à $15 \%$ en masse - et des hexa-aluminates de chaux [7]. Les phases formées améliorent la résistance à la corrosion [7,15] en limitant l'infiltration du laitier (constitué de chaux, d'alumine, d'oxyde de fer et de silice) et la résistance mécanique. On observe (Fig. 3) une zone plus sombre, pouvant atteindre plusieurs centimètres de profondeur, où la microstructure du matériau est modifiée par l'imprégnation de la dalle par le laitier. Elle est limitée en surface par une fine couche de laitier résiduel et en profondeur par la limite d'imprégnation fixée sans doute par une isotherme en dessous de laquelle, il n'existe plus de phase liquide et ou de diffusion d'espèces. Un réseau de fissures parallèles et perpendiculaires s'est développé dans cette zone pâteuse à haute température (1300 à $1650^{\circ} \mathrm{C}$ ) et plus fragile à basse température. Certaines fissures sont imprégnées par du métal et du laitier qui interagissent de manière complexe avec la surface nouvelle produite par l'apparition et l'extension des fissures. La corrosion se traduit ici par des phénomènes de dissolution de phase, transport ou diffusion vers l'intérieur du réfractaire et précipitation. On notera ici que les couplages entre transformations de phase et mécanique sont particulièrement importants.

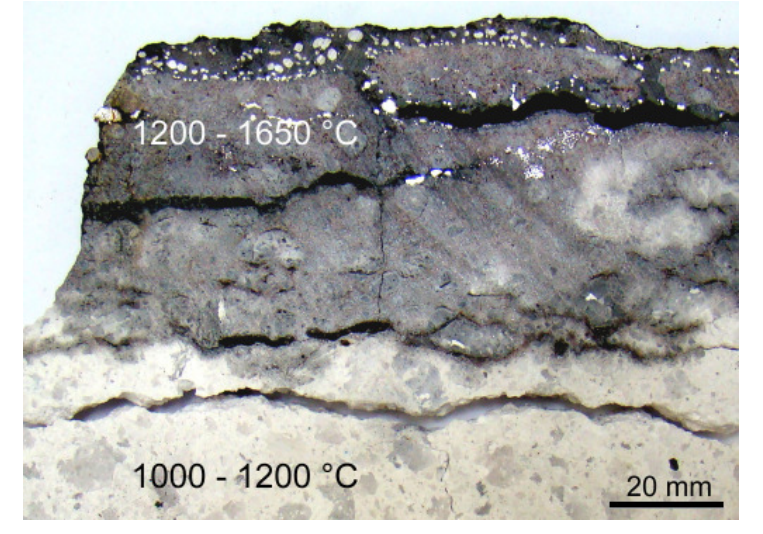

Fig. 3. Dégradation des dalles d'impact au contact des aciers et laitiers liquides [1]

Fig. 3. Degradations of impact pads in contact with liquid steel and slag [1]

\section{3) Quelques approches pour cerner les effets des transformations de phase sur la tenue mécanique des réfractaires}

Les exemples précédents ont illustré la complexité des phénomènes en jeu et l'analyse qu'il est nécessaire de développer pour cerner les causes d'endommagement des structures réfractaires. Les modèles numériques participent également à l'enrichissement de la connaissance sur ces phénomènes qui est principalement construite à partir d'observations microstructurales. L'étude des couplages multi-phénomène $[4,21]$ ou multi-échelle $[16,21]$ favorise le dialogue entre céramistes et mécaniciens et permettent d'apporter des éléments nouveaux pour l'analyse des dégradations.

\section{1) La simulation numérique de la microstructure, une voie pour comprendre le comportement mécanique local de la céramique}

L'exemple décrit ci-dessous est significatif de l'apport que constitue la simulation numérique de la microstructure pour mieux comprendre le comportement macroscopique des réfractaires observé à haute température.

Les réfractaires de type Alumine-Zircone-Silice (AZS) sont utilisés dans les fours verriers en raison de leur bonne résistance à la corrosion au contact des verres liquides et des fumées et au fluage à haute température (Fig. 4a) [16,18]. Pour la fabrication de certains verres spéciaux, on utilise des nuances à très forte teneur massique en zircone (THTZ) - typiquement voisine de $90 \%$ - pour limiter la corrosion de la phase alumine, silice. En l'absence d'effet lié à la corrosion, ces matériaux subissent principalement deux types de transformations de phase à haute température qui ont une incidence sur 
les propriétés mécaniques. Vers $800{ }^{\circ} \mathrm{C}$, la phase vitreuse se ramollit et devient très visqueuse. Elle peut donc se déformer aisément dans l'espace intergranulaire. La transformation martensitique bien connue de la zircone monoclinique en zircone tétraclinique se produit vers $1130^{\circ} \mathrm{C}$ au chauffage, la transformation inverse se produisant vers $1000^{\circ} \mathrm{C}$ au refroidissement. Les pièces réfractaires, élaborées à une température comprise entre 1900 et $2300^{\circ} \mathrm{C}$, s'endommagent au cours du refroidissement en raison de l'expansion volumique de la zircone (environ $4 \%$ ). Les moyens expérimentaux et numériques complémentaires mis en œuvre durant le programme ANR Promotheref [5] confirment le fait qu'il est aujourd'hui nécessaire de sonder les phénomènes à l'échelle de la microstructure si l'on souhaite améliorer la durée de vie de ces matériaux. A titre d'exemple, on présente sur la figure $4 \mathrm{~b}$ la caractérisation de la microstructure des AZS par tomographie X au synchrotron ESRF de Grenoble et des techniques d'analyse d'images et la modélisation numérique de cette structure par la méthode des éléments finis. Ces moyens associés à des moyens expérimentaux complémentaires ont permis aux acteurs associés au projet de mieux comprendre de quelle manière le comportement local affectait le comportement global de la structure. La morphologie très spécifique de la microstructure de ces réfractaires a été identifiée, leurs états des contraintes locales et résiduelles ont été estimés et les mécanismes d'endommagement et de cicatrisation possibles ont été identifiés. Le programme ANR a permis de développer un panel d'outils très prometteurs. De tels outils permettront à l'avenir de mieux optimiser le matériau en agissant simultanément sur certains effets antagonistes du comportement mécanique induits par ces transformations de phase. En effet, ceux-ci peuvent être soient pénalisantes (endommagement, diminution de la résistance au fluage) ou être bénéfiques (cicatrisation, refermeture de pores et de microfissures) selon le cas.

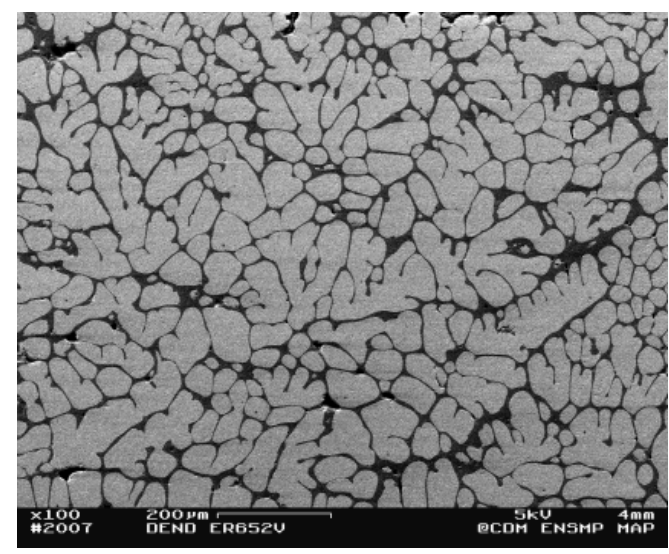

a) Microstructure d'un AZS à $24 \%$ de phase vitreuse [12]

a) Microstructure of an AZS refractory containing $24 \%$ of glassy phase [12] a

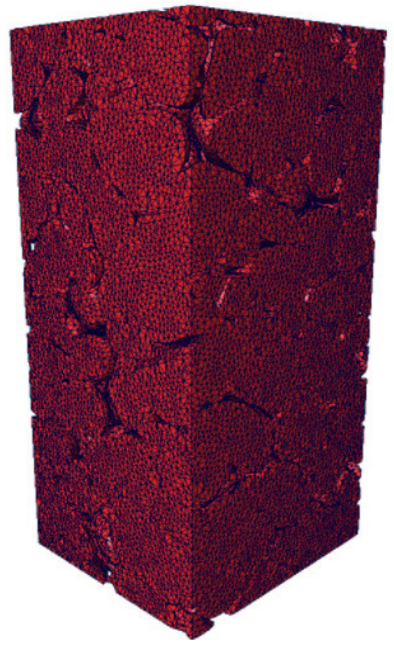

b) Modélisation de la microstructure déduite par tomographie X $(336 \mu \mathrm{m} \times 310 \mu \mathrm{m} \times 700 \mu \mathrm{m})[16]$

$b$ ) Modeling of a microstructure build from a X-ray tomography analysis of a specimen $(336 \mu \mathrm{m} \times 310 \mu \mathrm{m}$ $\times 700 \mu \mathrm{m})[16]$

Fig. 4. De l'observation de la microstructure des réfractaires électrofondus AZS vers la simulation numérique du comportement mécanique global du VER

Fig. 4. From microstructural observations of AZS fused refractories towards numerical simulations of the macroscopic behavior of REV

\section{2) Des modèles de thermo-chimie pour décrire les changements de phase dans les réfractaires}

L'analyse des phases présentes dans le réfractaire, en équilibre thermodynamique dans leur environnement, est essentiel pour comprendre les modifications du comportement mécanique observés in situ qu'on ne peut pas attribuer uniquement à un effet de la température. L'exemple ci-dessous illustre ce propos. 
Les réfractaires à base de magnésie carbone sont utilisés dans des garnissages d'outils sidérurgiques comme les convertisseurs et les poches de traitements métallurgiques secondaires de l'acier [23,25]. Leur microstructure est différente des AZS car la magnésie est présente sous forme de grains de taille au plus de quelques millimètres, enrobés d'un liant carboné contenant des additifs (quelques pour cent), notamment du graphite et des fines d'aluminium et de silicium (Fig. 5a). La magnésie a une température de fusion élevée mais est sensible au choc thermique. Le graphite présent dans la phase liante réduit la mouillabilité du réfractaire par les oxydes liquides et donc l'infiltration du laitier corrosif et améliore la résistance au choc thermique. Les particules ultrafines d'aluminium et de silicium améliorent la résistance à haute température du matériau (Fig. 5b) par la formation de carbure dès $600^{\circ} \mathrm{C}$ et la formation à haute température de spinelle primaire et secondaire comme le montre la figure $6 \mathrm{a}$.

Les réactions qui conduisent à ces transformations sont en réalité plus complexes que celles présentées sur la figure $6 \mathrm{~b}$. Les calculs thermodynamiques et l'exploitation des diagrammes de prédominance et de phases binaire et tertiaire, combinés aux observations microstructurales post-mortem ont permis de cerner les principaux mécanismes de transformation se produisant dans ces matériaux complexes [23,24]. L'utilisation d'outils de simulation thermo-chimie comme par exemple FactSage [2] permet de prendre en compte un plus grand nombre de phases et l'effet de l'environnement (température, pression partielle des gaz en contact avec les phases solides et liquides). Des explications plus détaillées peuvent ainsi être fournies sur les mécanismes de changement de phases en introduisant les étapes de transformations intermédiaires qui se succèdent. Elles permettent de franchir un pas supplémentaire dans la prise en compte de ces transformations dans un revêtement réfractaire dans lequel règne un gradient de température. Un exemple récent est présenté dans l'article de Berjonneau et al. [3]. L'intégration de ces informations dans un modèle de comportement mécanique reste encore un domaine ouvert. D'autres phénomènes non évoqués sont également observés dans ce matériau notamment la pyrolyse de la phase carbonée lors du premier chauffage et affectent notablement le comportement à haute température [14].

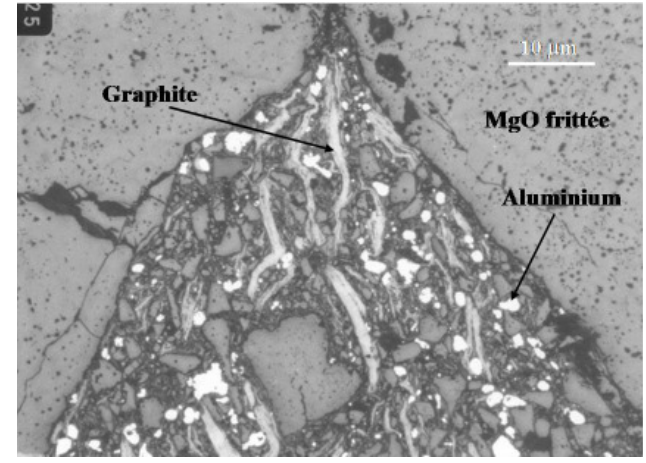

a) Microstructure d'une céramique de type magnésie graphite

a) Microstructure of a magnesia graphite ceramic

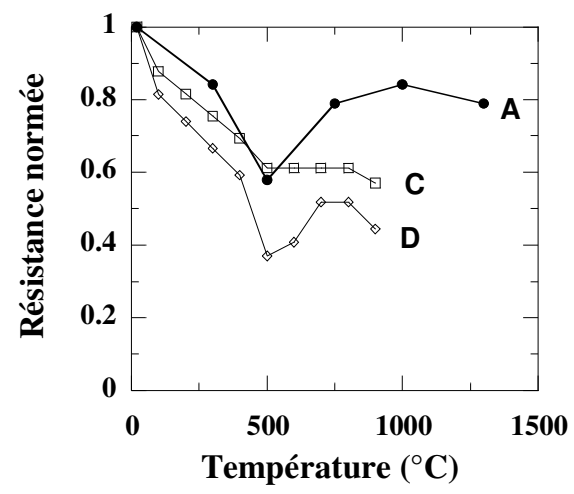

b) Evolution de la résistance en compression simple ; les nuances $\mathrm{A}$ et $\mathrm{D}$ possèdent des additifs, $\mathrm{C}$ ne contient pas d'additifs [6,20]

b) Change of compressive strength versus temperature; refractories $A$ and $D$ containing addition elements and $C$ not

Fig. 5. La maîtrise des propriétés mécaniques à haute température des réfractaires à base de magnésie carbone par l'ajout de fines d'aluminium

Fig. 5. Mastery mechanical properties of magnesia-carbon refractories at high temperature by additions of alumina small particles 

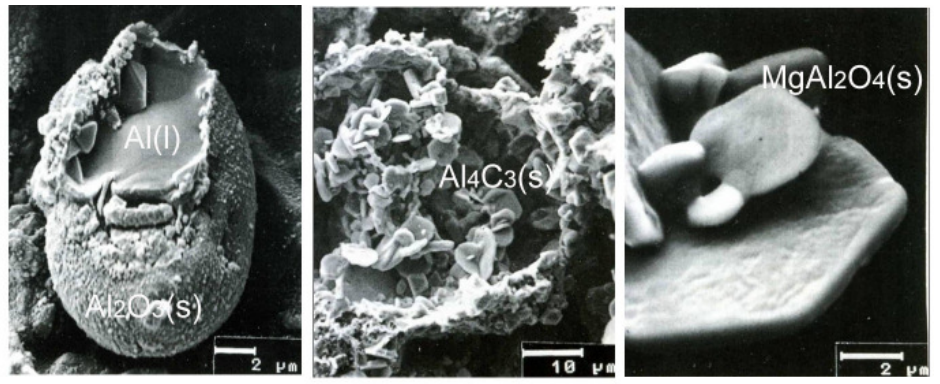

a) Transformations successives de l'aluminium (déchirure de l'enveloppe $\mathrm{Al}_{2} \mathrm{O}_{3}$ qui réagit avec $\mathrm{C}$ à $\mathrm{T}>660^{\circ} \mathrm{C}$ ), formation de carbure $\mathrm{Al}_{4} \mathrm{C}_{3}$ et de spinelle $\mathrm{Al}_{2} \mathrm{O}_{3}-\mathrm{MgO}$ à $\mathrm{T}>1100^{\circ} \mathrm{C}$

a) Progressive change of aluminum (break of $\mathrm{Al}_{2} \mathrm{O}_{3}$ skin reacting with $\mathrm{C}$ at $\mathrm{T}>660^{\circ} \mathrm{C}$ ), $\mathrm{Al}_{4} \mathrm{C}_{3}$ carbide and $\mathrm{Al}_{2} \mathrm{O}_{3^{-}}$ $\mathrm{MgO}$ spinel formation at $\mathrm{T}>1100^{\circ} \mathrm{C}$

$$
\begin{gathered}
4 \mathrm{Al}(\mathrm{l}, \mathrm{s})+3 \mathrm{C}(\mathrm{s}) \rightarrow \mathrm{Al}_{4} \mathrm{C}_{3}(\mathrm{~s}) \\
\mathrm{Al}_{4} \mathrm{C}_{3}(\mathrm{~s})+8 \mathrm{MgO}(\mathrm{s}) \rightarrow 2 \mathrm{MgAl}_{2} \mathrm{O}_{4}(\mathrm{~s})+ \\
3 \mathrm{C}(\mathrm{s})+6 \mathrm{Mg}(\mathrm{g}) \\
6 \mathrm{CO}(\mathrm{g})+\mathrm{Al}_{4} \mathrm{C}_{3}(\mathrm{~s}) \rightarrow 2 \mathrm{Al}_{2} \mathrm{O}_{3}(\mathrm{~s})+9 \mathrm{C}(\mathrm{s})
\end{gathered}
$$

b) Illustration de quelques réactions chimiques observées à haute température

c) Illustration of chemical reactions occurring at high temperature

Fig. 6. Observations microstructurales et identification des mécanismes de transformations à haute température dans les réfractaires à base de magnésie carbone

Fig. 6. Microstructure observations and identification of phase change mechanisms at high temperature in magnesia-carbon refractories

\section{3) Vers une modélisation des couplages multi-physique et multi-échelle des réfractaires}

L'exemple du séchage des réfractaires façonnés présenté ci-dessous démontre qu'il est possible d'intégrer les effets induits par les transformations de phase sur le comportement mécanique du VER (volume équivalent représentatif) du réfractaire dans une modélisation prédictive du comportement de structure.

La vitesse de déshydratation de la phase liante du béton réfractaire alumineux qui n'est que partiellement hydratée doit être contrôlée pour éviter que des surpressions de vapeur excessives ne détruisent le béton. Ce séchage nécessite de bien maîtriser les paramètres «matériau ». Un modèle d'homogénéisation spécifique a été développé dans ce but [21]. A l'échelle du grain hydraté, la cinétique de perte de masse d'eau lors d'un séchage a été identifiée en considérant en première approximation que les grains hydratés (gybbsite $\mathrm{AH}_{3}$ et hexa-aluminate de calcium hydraté $\mathrm{C}_{3} \mathrm{AH}_{6}$ où A, $\mathrm{C}$ et $\mathrm{H}$ désignent respectivement $\mathrm{Al}_{2} \mathrm{O}_{3}, \mathrm{CaO}$ et $\mathrm{H}_{2} \mathrm{O}$ ) se déshydratent en suivant la topologie précisée sur la figure 7a, ce qui conduit à identifier quatre cinétiques de décomposition. Un exemple de résultat d'identification par des analyses thermogravimétriques ATG est présenté sur la figure 8a. Les taux de conversion d'hydrates sont ensuite introduits dans un modèle multi-échelle complexe dont on ne montre sur la figure $7 \mathrm{~b}$ que la partie homogénéisation de la phase liante - elle-même obtenue par une autre homogénéisation - combinée aux grains d'alumine. La qualité de la prédiction des propriétés macroscopiques est montrée sur les figures $8 \mathrm{~b}$ et $8 \mathrm{c}$. Cette approche se révèle intéressante car d'une part elle relie des propriétés mécaniques du matériau à des paramètres de composition chimique et minéralogique du réfractaire et un état de transformation et, d'autre part, elle sert de référence pour identifier un modèle de déshydratation simplifié qui peut être introduit dans un calcul de structure comme cela a été réalisé dans [22] permettant ainsi de déterminer les contraintes développées dans un revêtement de durée d'une poche à acier en phase de séchage et de proposer des solutions pour limiter le risque de fissuration (voir Fig. 1). 


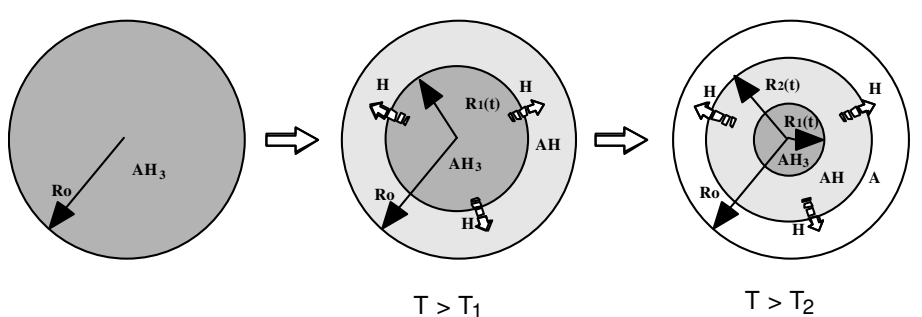

a) Topologie simplifiée : cas de la cinétique de déshydratation de $\mathrm{AH}_{3}\left(\mathrm{~A}=\mathrm{Al}_{2} \mathrm{O}_{3}\right.$ et $\left.\mathrm{H}=\mathrm{H}_{2} \mathrm{O}\right)$

a) Simplified topology: example of the dehydration kinetics of $\mathrm{AH}_{3}\left(\mathrm{~A}=\mathrm{Al}_{2} \mathrm{O}_{3}\right.$ et $\left.\mathrm{H}=\mathrm{H}_{2} \mathrm{O}\right)$

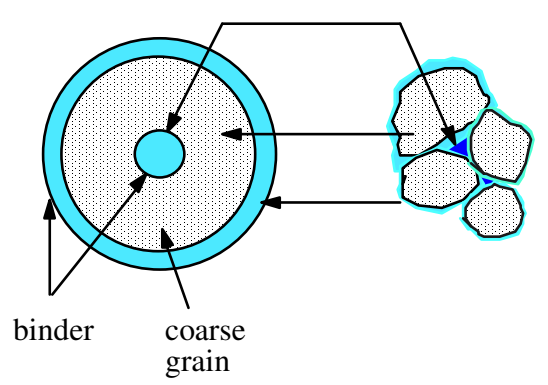

b) Homogénéisation du béton réfractaire utilisant le modèle de Larrard et le Roy

b) Homogenization of the concrete using the de Larrard et le Roy model

Fig. 7. Un modèle multi-échelle et multi-physique pour le séchage des bétons réfractaires [21]

Fig. 7. A multi-scale and multi-physical model for the drying of refractory concretes [21]

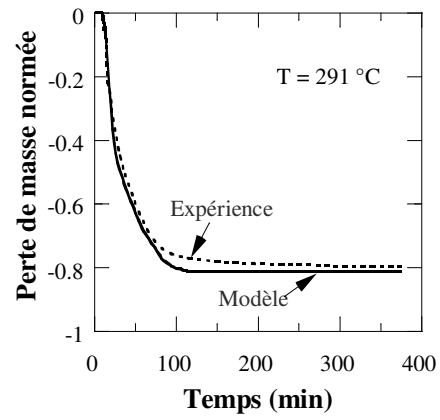

a) Identification de la cinétique de déshydratation du liant hydraulique

a) Identification of the dehydration kinetics of hydraulic binder

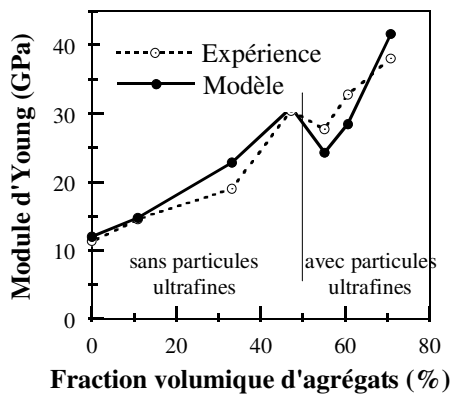

b) Estimation du module d'Young macroscopique du réfractaire

b) Estimate of the macroscopic Young's modulus of the refractory

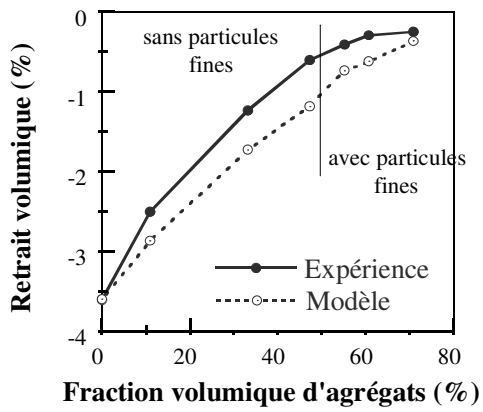

c) Estimation du retrait de déshydratation macroscopique du réfractaire

c) estimate of the dehydration shrinkage of the refractory

Fig. 8. Confrontation expérience et modèle multi-échelle et multiphysique [21]

Fig. 8. Comparison between experimental data and the multi-scale and multi-physical model [21]

\section{Conclusion}

Les études sur la tenue en service des céramiques réfractaires font aujourd'hui de plus en plus souvent appel à des méthodes combinant des analyses expérimentales et numériques complémentaires faisant intervenir les phénomènes d'origine variée pour identifier les causes des dégradations et de changement de leur comportement thermomécanique. Les cas de dégradation de structures réfractaires (sections $\S 2$ et $\S 3$ ) s'inscrivent dans un schéma composé de quatre grands pôles, i.e. «ThermiqueMécanique-Transfert-Changement de phase » qui sont reliés entre eux par des couplages plus ou moins forts. Une analyse fine de ces couplages permet alors de sélectionner les moyens expérimentaux et numériques les plus appropriés.

Ces études sont possibles en raison des progrès dans les techniques de caractérisation de la microstructure et notamment des nouvelles méthodes numériques permettant d'aborder les nombreux changements d'échelle qu'il est nécessaire de prendre en compte pour relier le comportement du matériau à l'échelle micrométrique au comportement du matériau à l'échelle macroscopique 
$[4,9,16,21]$. Ces outils offrent à la communauté scientifique travaillant dans le domaine des matériaux réfractaires de nouvelles opportunités pour mener des études pluridisciplinaires en vue d'améliorer la connaissance et la prédiction des phénomènes de dégradations des structures réfractaires. Pour les industries utilisatrices de ces produits réfractaires, de nouvelles perspectives se dégagent également grâce à l'apparition sur le marché des réfractaires plus performants qui permettent de réduire le coût « réfractaire » - il faut garder à l'esprit que la consommation réfractaire dans l'industrie sidérurgique est supérieure à $10 \mathrm{~kg} /$ tonne d'acier produit. Mais surtout, l'utilisation de réfractaires plus durables dans des environnements plus sévères (températures plus élevées, environnement «chimique » plus agressif) autorise la mise sur la marché de produits manufacturés nouveaux et de meilleure qualité (e.g., de nuances d'acier nouvelles ou pointues: à haut Mn, HLE, TRIP, DWI, ...)

\section{Bibliographie}

1 Arfan E. et al., Surface damage of alumina-spinel castable due to thermal shock by liquid metal : a numerical analysis, Proc. $2^{\text {nd }}$ Int. Cong. Ceramics, June 29-July 4, Verona (Italy), 2008.

2 Bale C.W. et al., FactSage thermochemical software and databases, Calphad, 26 [2], 189-228, 2002

3 Berjonneau J., Prigent, P., Poirier J., The development of a thermodynamic model for $\mathrm{Al}_{2} \mathrm{O}_{3}-\mathrm{MgO}$ refractory castable corrosion by secondary metallurgy steel ladle slags, Ceramics International, 35 [2] 623-635, (2009)

4 Blond E. et al., Effect of slag impregnation on thermal degradations in refractories, J. Am. Ceram. Soc., 90 [1], 154-162, 2007

5 Boussuge M., Investigation of the thermomechanical properties of industrial refractories : the French programme PROMETHEREF, J. Mater. Sci., 43, 4069-4078, 2008

6 Fitchett A.M. et B. Wilshire B., Mechanical properties of carbon-bearing magnesia, Part I, II and III, Br. Ceram. Trans. J., 1984, 83, 54-76.

7 Führer M., Hey A., Lee W.E., Microstructural evolution in self forming spinel calcium aluminate bonded castable refractories , J. Eur. Ceram. Soc., 18, 813-820, 1998

8 Gault C. et al., Le programme DRuIDE : présentation et premiers résultats, Journées spécialisées sur les réfractaires, 23-24 septembre 2008, Lunéville (France), 13-16, 2008

9 Gasser A. et al., Thermomechanical behaviour analysis and simulation of steel/refractory composite linings, Composites Sci. Tech., 61, 2095-2100, 2001.

10 Karakus M. et Moore R.E., J. Min. \& Mat. Characterization \& Eng., 1 [1], 11-29, 2002

11 Korgul P., Wilson D.R., Lee W.E., Microstructural analysis of corroded alumina-spinel castable refractories, J. Eur. Ceram. Soc., 17, 77-84 (1997)

12 Lastate E. et al., Microstructural and mechanical consequences of thermal cycles on a high zirconia fuse-cast refractory, J. Eur. Ceram. Soc., 29, 587-594, 2009

13 Lee W.E. et Moore R.E., Evolution of in Situ Refractories in the 20th Century, J. Am. Ceram. Soc., 81 [6], 1385-1410, 1998

14 Lubaba N.C. et al., Effect of carbon binders on the development of porosity in MgO-Graphite composite refractories, Br. Ceram. Trans. J., 87, 164-167, 1988

15 Ganesh I. et al., Ceramics International, 28, 245-253, 2002

16 Madi K. et al., Finite element simulations of the deformation of fused-cast refractories based on X-ray computed tomography, Computational Materials Science, 39, 224-229, 2007

17 Marzagui H. et Cutard T., Characterisation of microstructural evolutions in refractory castables by in situ high temperature ESEM, J. Mat. Proc. Tech., 155-156, 1474-1481, 2004

18 Poirier J., Chap. 3, propriétés et Applications des céramiques réfractaires, Traité MIM, série alliages métalliques), Pub. Ph. Boch, Ed. Hermes Science, Paris, 2001 
19 Prigent P., Approche multi-échelle des mécanismes de corrosion à haute température des céramiques réfractaires, Thèse de doctorat, Polytech'Orléans, en cours

20 Robin J.M. et al., Thermomechanical behaviour of magnesia-carbon refractories, Br. Ceram. Trans. J., 97 [1],1-10, 1998

21 Schmitt N. et al., Coupling between kinetics of dehydration, physical and mechanical behaviour for high alumina castable., Cem. Concrete Res., 30, 1597-1607, 2000

22 Schmitt N. et al., Damage of monolithic refractory linings in steel ladles during drying, $\mathrm{Br}$. Ceram. Trans. J., 103 [3], 121-133, 2004

23 Taffin C. et Poirier J., Behaviour of metal additives in $\mathrm{MgO}-\mathrm{C}$ and $\mathrm{Al}_{2}-\mathrm{O}_{3}-\mathrm{C}$ refractories, Interceram., 43, 458-460, 1994

24 Uchida S. et al., High-temperature properties of unburned $\mathrm{MgO}-\mathrm{C}$ bricks containing $\mathrm{Al}$ and $\mathrm{Si}$ powders, J. Am. Ceram. Soc., 81 [11], 2910-2916, 1998

25 Yamaguchi A., Thermomechanical analysis for reaction processes of aluminium - compounds in carbon - containing refractories, Taikabutsu, 35, 617-622, 1983 